Mathematical Modelling And AnAlysis

Volume 20 Number 5, September 2015, 552-577

http://dx.doi.org/10.3846/13926292.2015.1085921

(c) Vilnius Gediminas Technical University, 2015
Publisher: Taylor\&Francis and VGTU

http://www.tandfonline.com/TMMA

ISSN: $1392-6292$

eISSN: $1648-3510$

\title{
Strong Stability Preserving Multistage Integration Methods*
}

\author{
Giuseppe $\operatorname{Izzo}^{a}$ and Zdzislaw Jackiewicz ${ }^{b, c}$ \\ ${ }^{a}$ Dipartimento di Matematica e Applicazioni, Università di Napoli- \\ "Federico II" \\ 80126 Napoli, Italy \\ ${ }^{b}$ Department of Mathematics, Arizona State University \\ Tempe, 85287 AZ, United States \\ ${ }^{c}$ AGH University of Science and Technology \\ Kraków, Poland \\ E-mail(corresp.): giuseppe.izzo@unina.it \\ E-mail: jackiewicz@asu.edu
}

Received September 30, 2014; revised July 14, 2015; published online September 15, 2015

\begin{abstract}
In this paper we systematically investigate explicit strong stability preserving (SSP) multistage integration methods, a subclass of general linear methods (GLMs), of order $p$ and stage order $q \leq p$. Characterization of this class of SSP GLMs is given and examples of SSP methods of order $p \leq 4$ and stage order $q=1,2, \ldots, p$ are provided. Numerical tests are reported which confirm that the constructed methods achieve the expected order of accuracy and preserve monotonicity.
\end{abstract}

Keywords: multistage integration methods, general linear methods, strong stability preserving, monotonicity, two-step Runge-Kutta methods.

AMS Subject Classification: 65L06; 65L20; 65L99.

\section{Introduction}

Many practical problems in sciences and engineering are modeled by systems of ordinary differential equations (ODEs) which arise from semidiscretization of partial differential equations (PDEs) of mathematical physics. Such systems take the form

$$
\left\{\begin{array}{l}
y^{\prime}(t)=f(t, y(t)), \quad t \in\left[t_{0}, t_{\text {end }}\right], \\
y\left(t_{0}\right)=y_{0}
\end{array}\right.
$$

where the function $f: \mathbb{R} \times \mathbb{R}^{m} \rightarrow \mathbb{R}^{m}$ is assumed to have some degree of smoothness.

\footnotetext{
* The work of the first author (GI) was partially supported by GNCS-INdAM.
} 
We assume that the discretization of (1.1) by the forward Euler method

$$
y_{n}=y_{n-1}+h f\left(t_{n-1}, y_{n-1}\right),
$$

$n=1,2, \ldots, N, N h=t_{\text {end }}-t_{0}, t_{n}=t_{0}+n h$, is monotone or contractive. This means that the following inequality holds

$$
\left\|y_{n}\right\| \leq\left\|y_{n-1}\right\|
$$

$n=1,2, \ldots, N$, in some norm or semi-norm $\|\cdot\|$, for a suitably restricted time step determined by the condition $h \leq h_{F E}$.

In the class of multistage integration methods we want to determine higher order numerical methods for (1.1), which preserve the monotonicity property (1.2), under the restriction

$$
h \leq \mathcal{C} h_{F E} .
$$

Numerical schemes for (1.1) that preserve the monotonicity condition (1.2) under the modified restriction (1.3) are called strong stability preserving (SSP) methods and the constant $\mathcal{C} \geq 0$ in (1.3) is called SSP coefficient.

SSP Runge-Kutta (RK) and linear multistep methods (LMMs) have been first studied, using the terminology total variation diminishing (TVD) time discretizations, by Shu and Osher [50]. Then, they were further investigated by Gottlieb et al. [22, 23, 24, 25, 27, 43], Spiteri and Ruuth [53], Hundsdorfer and Ruuth [32], Hundsdorfer, Ruuth and Spiteri [33], Higueras [28, 29,30,31] and Ferracina and Spijker $[18,19,20,21]$. SSP two-step Runge-Kutta (TSRK) methods, introduced by Jackiewicz and Tracogna [40], were investigated by Ketcheson, Gottlieb and Macdonald in [42]. Constantinescu and Sandu [8] generalized Shu-Osher representation to a class of multistep multistage schemes, which form a special subclass of GLMs. SSP GLMs were investigated by Spijker [52] and by Izzo and Jackiewicz [36,37].

In this paper we systematically investigate a subclass of explicit GLMs in order to derive methods with optimal SSP coefficient $\mathcal{C}$.

It is known that irreducible strong stability preserving Runge-Kutta methods have positive stage coefficients, $a_{i j} \geq 0$, and strictly positive weights, $b_{j}>0$. A similar property holds for SSP two-step Runge-Kutta methods [23]. Gottlieb and Shu [26] proved that no four-stage, fourth-order SSPRK method exists having positive coefficients, while Ruuth and Spiteri [47] proved that an SSPRK method with positive coefficients and order $p>4$ cannot exist (for general nonlinear problems). The class of general linear methods allows to break these two barriers and it also permits to construct methods with high stage order.

The paper is organized as follows: in Section 2 we report the formulation of general linear methods (GLMs), the related order conditions and a criterion to determine the SSP coefficient $\mathcal{C}$. In Sections 3 and 4 we characterize some classes of explicit SSP multistage integration methods of order $p$ and stage order $q \leq p$ and investigate them to construct methods with a large SSP coefficient. In Section 5 we describe the starting and finishing procedures, while in Section 6 we present the results of numerical experiments to validate order and monotonicity preservation of the new SSP schemes. Finally, in Section 7 some concluding remarks are given and plans for future research are briefly outlined. 


\section{SSP Conditions for GLMs}

\subsection{General linear methods}

General linear methods $[1,2,3,39]$ for the numerical solution of ODEs (1.1) are defined by

$$
\begin{cases}Y_{i}^{[n]}=h \sum_{j=1}^{s} a_{i j} f\left(t_{n-1}+c_{j} h, Y_{j}^{[n]}\right)+\sum_{j=1}^{r} u_{i j} y_{j}^{[n-1]}, & i=1,2, \ldots, s, \\ y_{i}^{[n]}=h \sum_{j=1}^{s} b_{i j} f\left(t_{n-1}+c_{j} h, Y_{j}^{[n]}\right)+\sum_{j=1}^{r} v_{i j} y_{j}^{[n-1]}, & i=1,2, \ldots, r,\end{cases}
$$

$n=1,2, \ldots, N$, where $N h=t_{\text {end }}-t_{0}$. In (2.1) the internal approximations or stages approximate the solution $y$ to (1.1) at the points $t_{n-1}+c_{i} h$ to the stage order $q$, i.e.,

$$
Y_{i}^{[n]}=y\left(t_{n-1}+c_{i} h\right)+O\left(h^{q+1}\right), \quad i=1,2, \ldots, s,
$$

and the external approximations $y_{i}^{[n]}$ approximate the linear combinations of the scaled derivatives of the solution at the grid point $t_{n}$ to the order $p$, i.e.,

$$
y_{i}^{[n]}=\sum_{k=0}^{p} q_{i k} h^{k} y^{(k)}\left(t_{n}\right)+O\left(h^{p+1}\right), \quad i=1,2, \ldots, r .
$$

These methods are specified by the abscissa vector $\mathbf{c}=\left[c_{1}, \ldots, c_{s}\right]^{T} \in \mathbb{R}^{s}$, four coefficient matrices

$$
\mathbf{A}=\left[a_{i j}\right] \in \mathbb{R}^{s \times s}, \quad \mathbf{U}=\left[u_{i j}\right] \in \mathbb{R}^{s \times r}, \quad \mathbf{B}=\left[b_{i j}\right] \in \mathbb{R}^{r \times s}, \quad \mathbf{V}=\left[v_{i j}\right] \in \mathbb{R}^{r \times r},
$$

the vectors $\mathbf{q}_{i}=\left[q_{1, i}, \ldots, q_{r, i}\right]^{T} \in \mathbb{R}^{r}, i=0,1, \ldots, p$, and four integers: the order of the method $p$, the stage order $q$, the number of external approximations $r$, and the number of internal approximations or stages $s$.

The framework of general linear methods have been used recently to analyze the numerical stability of several class of methods $[4,5,9,10,11,13,14,15]$. General linear methods for second order equations were considered in $[12,16,17]$ and for VIEs and VIDEs in $[38,54]$.

\subsection{Order conditions for GLMs}

The order conditions for GLMs (2.1) of order $p \leq 4$ are reported in Table 1 (compare Butcher [3], Jackiewicz and Vermiglio [41], Cardone et. al. [6]), where

$$
\begin{aligned}
& \gamma_{0}=\mathbf{e}-\mathbf{U} \mathbf{q}_{0}, \quad \gamma_{k}=\frac{\mathbf{c}^{k}}{k !}-\frac{\mathbf{A} \mathbf{c}^{k-1}}{(k-1) !}-\mathbf{U} \mathbf{q}_{k}, \quad k=1,2, \ldots, p, \\
& \widehat{\gamma}_{0}=\mathbf{q}_{0}-\mathbf{V} \mathbf{q}_{0}, \quad \widehat{\gamma}_{k}=\sum_{l=0}^{k} \frac{\mathbf{q}_{l}}{(k-l) !}-\frac{\mathbf{B c}^{k-1}}{(k-1) !}-\mathbf{V} \mathbf{q}_{k}, \quad k=1,2, \ldots, p,
\end{aligned}
$$


Table 1. Order conditions for GLMs (2.1) of order $p \leq 4$.

\begin{tabular}{cc}
\hline Order & Corresponding order conditions \\
\hline$p=1$ & $\widehat{\gamma}_{1}=0$ \\
\hline$p=2$ & $\widehat{\gamma}_{2}=0$ \\
\hline$p=3$ & $\widehat{\gamma}_{3}=0$ \\
& $\mathbf{V B} \gamma_{2}=0$ or $\mathbf{B} \gamma_{2}=0$ \\
\hline$p=4$ & $\widehat{\gamma}_{4}=0, \quad \mathbf{V B} \gamma_{3}=0$ \\
& $\mathbf{V B A} \gamma_{2}=0, \quad \mathbf{V B} \boldsymbol{\Gamma}_{\mathbf{c}} \gamma_{2}=0$ \\
\hline
\end{tabular}

and $\boldsymbol{\Gamma}_{\mathbf{c}}=\operatorname{diag}\left(c_{1}, \ldots, c_{s}\right), \mathbf{e}=[1,1, \ldots, 1]^{T} \in \mathbb{R}^{s}$. Let us remark that stage order $q$ can be achieved by forcing $\gamma_{k}=0$ for $k=0,1, \ldots, q$.

It will be always assumed that

$$
\sum_{j=1}^{r} v_{i j}=1, \quad i=1,2, \ldots, r
$$

and $\mathbf{q}_{0}=[1,1, \ldots, 1]^{T} \in \mathbb{R}^{r}$, so that the stage preconsistency condition $\gamma_{0}=0$, or $\mathbf{U} \mathbf{q}_{0}=\mathbf{e}$, and the preconsistency condition $\widetilde{\gamma}_{0}=0$, or $\mathbf{V} \mathbf{q}_{0}=\mathbf{q}_{0}$, are automatically satisfied (compare [39]). When in Table 1 there is a couple of conditions separated by 'or', the first condition refer to order $p$ methods, while the second condition refers to methods with order greater than $p$.

\subsection{Characterization of SSP coefficient for GLMs}

Following [37] we consider the componentwise inequalities

$$
\begin{gathered}
(\mathbf{I}+\gamma \mathbf{A})^{-1} \mathbf{U} \geq 0, \quad \mathbf{I}-(\mathbf{I}+\gamma \mathbf{A})^{-1} \geq 0, \\
\mathbf{V}-\gamma \mathbf{B}(\mathbf{I}+\gamma \mathbf{A})^{-1} \mathbf{U} \geq 0, \quad \gamma \mathbf{B}(\mathbf{I}+\gamma \mathbf{A})^{-1} \geq 0 .
\end{gathered}
$$

It was demonstrated in [37] using the results of Spijker [52] that the SSP coefficient $\mathcal{C}$ of GLMs (2.1) can be characterized as

$$
\mathcal{C}=\mathcal{C}(\mathbf{c}, \mathbf{A}, \mathbf{U}, \mathbf{B}, \mathbf{V})=\sup \{\gamma \in \mathbb{R}: \gamma \text { satisfies }(2.3)\} .
$$

Similarly as in [42] this coefficient $\mathcal{C}$ can be computed, by the solution to the constrained minimization problem

$$
\min _{\gamma, \mathbf{c}, \mathbf{A}, \mathbf{U}, \mathbf{B}, \mathbf{V}, \mathbf{q}_{1}, \ldots, \mathbf{q}_{p}}-\gamma,
$$

subject to inequality constraints (2.3) and equality constraints

$$
\Phi_{p, q}\left(\mathbf{c}, \mathbf{A}, \mathbf{U}, \mathbf{B}, \mathbf{V}, \mathbf{q}_{1}, \ldots, \mathbf{q}_{p}\right)=0,
$$

where $\Phi_{p, q}$ represents the order conditions up to the order $p$ and stage order conditions up to the stage order $q \leq p$. 
In order to compare methods with different number of stages $s$ we also define, as in [8] and [42], the effective SSP coefficient by the normalization $\mathcal{C}_{\text {eff }}=\mathcal{C} / s$.

Izzo and Jackiewicz [37] investigated the strong stability preserving property for a subclass of explicit general linear methods of order $p=2,3,4$, stage order $q=1,2, \ldots, p$, with $r=2,3$ external stages and $s=2,3, \ldots, 10$ internal stages. Methods investigated in [37] contain the class of diagonally implicit multistage integration methods (DIMSIMs), the class of methods of order $p$ and stage order $q=p$ or $q=p-1$, with $s$ internal stages and $r=s$ or $r=s+1$ external stages, and rank one matrix $\mathbf{V}$.

In this paper we systematically investigate the SSP property for a subclass of explicit GLMs of order $p$ and stage order $q=1,2, \ldots, p$, characterized by having the number of external stages $r$ and the number of internal stages $s$, which equal the order of the method $p$ (i.e. $p=r=s$ ), and no restriction on the rank of $\mathbf{V}$ is forced. Hereafter we will generally refer to this subclass as multistage integration methods.

\section{Methods with $p=r=s$ and $\mathbf{U}=\mathbf{I}$}

In this section we systematically investigate explicit multistage integration methods of order $p \leq 4$ and such that

$$
\mathbf{U}=\mathbf{I}, \quad \text { and } \quad p=r=s, \quad q=1,2, \ldots, p .
$$

To characterize this class of methods we will need the following lemma.

Lemma 1. For an explicit SSP GLM satisfying (3.1) the matrix A must be identically equal to the zero matrix.

Proof. Let $\mathbf{L}=\left[l_{i j}\right]_{i, j=1}^{s}$ be the matrix given by $\mathbf{L}=(\mathbf{I}+\gamma \mathbf{A})^{-1}$. Since $\mathbf{A}$ is strictly lower triangular, $\mathbf{L}$ is a lower triangular matrix such that $l_{i i}=1$, $i=1,2, \ldots, s$. Furthermore,

$$
\mathbf{L}=(\mathbf{I}+\gamma \mathbf{A})^{-1}=\mathbf{I}-\gamma \mathbf{A}+\gamma^{2} \mathbf{A}^{2}+\cdots+(-\gamma \mathbf{A})^{s-1}=\sum_{i=0}^{s-1}(-\gamma \mathbf{A})^{i},
$$

and we have $\operatorname{tril}(\mathbf{L})=\sum_{i=1}^{s-1}(-\gamma \mathbf{A})^{i}$, where $\operatorname{tril}(\mathbf{L})$ stands for the strictly lower triangular part of $\mathbf{L}$. Let us consider the two SSP conditions

$$
\mathbf{L U} \geq 0, \quad \mathbf{I}-\mathbf{L} \geq 0 .
$$

If $\mathbf{U}=\mathbf{I} \in \mathbb{R}^{s \times s}$, from (3.2) we have $0 \leq \mathbf{L} \leq \mathbf{I}$, hence $\operatorname{tril}(\mathbf{L}) \equiv 0$, so that

$$
\sum_{i=1}^{s-1}(-\gamma \mathbf{A})^{i} \equiv 0
$$

Since matrix $\mathbf{A}$ is strictly lower triangular, from (3.3) we have $\gamma a_{i+1, i}=0$, for $i=1,2, \ldots, s-1$. Hence for SSP methods with $\gamma>0$ we have

$$
a_{i+1, i}=0, \quad i=1,2, \ldots, s-1 .
$$


From (3.3) and (3.4) we also obtain

$$
a_{i+2, i}=0, \quad i=1,2, \ldots, s-2 .
$$

Using induction it can be easily proved that

$$
a_{i j}=0, \text { for } i, j=1,2, \ldots, s .
$$

Remark. As consequence of Lemma 1, for GLMs satisfying (3.1), the stage order conditions $\gamma_{k}=0, k=0, \ldots, q$, lead to

$$
\mathbf{q}_{k}=\frac{\mathbf{c}^{k}}{k !}, \quad k=0, \ldots, q,
$$

and the SSP constraints (2.3) reduce to

$$
\mathbf{0} \leq \gamma \mathbf{B} \leq \mathbf{V} .
$$

Equations (2.3) and (3.5) imply that $0 \leq v_{i j} \leq 1$ for all $i, j=1,2, \ldots, s$, and, when $\gamma$ is positive, $0 \leq b_{i j} \leq 1$ for all $i, j=1,2, \ldots, s$. This information is helpful to set sharper (lower and upper) bounds on the parameters in the minimization process to solve the problem (2.5).

For SSP methods, from (3.5) we obtain $b_{i j} \geq 0$ for all $i, j=1,2, \ldots, s$ and

$$
0 \leq \gamma \leq \frac{v_{i j}}{b_{i j}}, \quad \text { for } i, j=1,2, \ldots, s, \text { and } b_{i j} \neq 0 .
$$

Since (3.5) are the only constraints, we obtain

$$
\mathcal{C}=\min \left\{\frac{v_{i j}}{b_{i j}}: i, j=1,2, \ldots, r, \text { and } b_{i j} \neq 0\right\} .
$$

In addition, (3.5) implies

$$
\mathbf{0} \leq \gamma \mathbf{B e} \leq \mathbf{V e}=\mathbf{e}
$$

and hence, for an SSP GLM satisfying (3.1) with SSP coefficient $\mathcal{C}$, the following necessary condition must hold

$$
\mathcal{C} \leq 1 / \sum_{j=1}^{s} b_{i j} \quad \text { and } \quad \sum_{j=1}^{s} b_{i j}>0, \quad i=1,2, \ldots, s .
$$

Furthermore, from order condition for $p=1$

$$
\mathbf{q}_{0}+\mathbf{q}_{1}-\mathbf{B e}-\mathbf{V} \mathbf{c}=0
$$

we obtain

$$
\mathrm{Be}=\mathbf{e}+\mathbf{c}-\mathbf{V} \mathbf{c} .
$$

This leads to

$$
0 \leq \sum_{j=1}^{s} b_{i j}=1+c_{i}-\sum_{j=1}^{s} v_{i j} c_{j}, \quad i=1,2, \ldots, s .
$$

Hence the necessary condition (3.7) can be also written as

$$
\mathcal{C} \leq \frac{1}{1+c_{i}-\sum_{j=1}^{s} v_{i j} c_{j}} \quad \text { and } \quad 1+c_{i}-\sum_{j=1}^{s} v_{i j} c_{j}>0, \quad i=1,2, \ldots, s .
$$


3.1 Methods with $p=r=s=2$

Consider the multistage integration method with coefficients

$$
\begin{aligned}
& {\left[\begin{array}{l|l}
\mathbf{A} & \mathbf{U} \\
\hline \mathbf{B} & \mathbf{V}
\end{array}\right]=\left[\begin{array}{cc|cc}
0 & 0 & 1 & 0 \\
a_{21} & 0 & 0 & 1 \\
\hline b_{11} & b_{12} & v_{1} & 1-v_{1} \\
b_{21} & b_{22} & v_{2} & 1-v_{2}
\end{array}\right], \quad \mathbf{c}=\left[\begin{array}{ll}
c_{1} & c_{2}
\end{array}\right]^{T},} \\
& \mathbf{q}_{\mathbf{0}}=\left[\begin{array}{ll}
1 & 1
\end{array}\right]^{T}, \quad \mathbf{q}_{1}=\left[\begin{array}{ll}
q_{11} & q_{21}
\end{array}\right]^{T}, \quad \mathbf{q}_{2}=\left[\begin{array}{ll}
q_{12} & q_{22}
\end{array}\right]^{T} .
\end{aligned}
$$

\subsubsection{Methods with $p=r=s=2, q=2$}

Solving the order conditions for order $p=2$ and stage order $q=2$ we obtain a 5-parameter family depending on $c_{1}, c_{2}, v_{1}, v_{2}$ and $a_{21}$ under the restriction $\left|v_{1}-v_{2}\right|<1$, which is equivalent to the power boundedness of the matrix $\mathbf{V}$, and hence it ensure the zero-stability of the method (compare [39]). Furthermore, from Lemma 1 we obtain $a_{21}=0$.

Case $v_{1}=v_{2}(\operatorname{rank}(\mathbf{V})=1)$.

Maximizing the SSP coefficient $\mathcal{C}$, adding the restriction $c_{1}<c_{2}$, we obtain:

$$
\mathcal{C}=1-\frac{1}{\sqrt{2}} \quad \text { for } c_{1}=0, c_{2}=\sqrt{2} \text { and } v_{1}=\frac{1}{2} .
$$

Case $v_{1} \neq v_{2}(\operatorname{rank}(\mathbf{V})=2)$.

Maximizing the $\mathrm{SSP}$ coefficient $\mathcal{C}$, adding the restriction $-1 \leq c_{1}<c_{2} \leq 1$, we obtain:

$$
\mathcal{C}=\frac{1}{2} \quad \text { for } c_{1}=-1, c_{2}=1, v_{1}=\frac{53}{64} \text { and } v_{2}=\frac{1}{4},
$$

while, adding the restriction $-2 \leq c_{1}<c_{2} \leq 2$, we obtain:

$$
\mathcal{C}=\frac{3}{4} \quad \text { for } c_{1}=-2, c_{2}=2, v_{1}=\frac{243}{256} \text { and } v_{2}=\frac{1}{16} .
$$

Choosing larger bounds for $c_{1}$ and $c_{2}$, the value of $\mathcal{C}$ increases (up to 1 ) but unfortunately also the magnitude of the $q$ 's increases. Our numerical search points out that in this case the best attainable value for $\mathcal{C}$ seems to be

$$
\mathcal{C}=1-\frac{1}{c_{2}-c_{1}}
$$

For the values given by (3.9) the method has the coefficients:

$$
\begin{gathered}
\mathbf{B}=\left[\begin{array}{cc}
\frac{37}{64} & \frac{5}{64} \\
0 & \frac{3}{2}
\end{array}\right], \quad \mathbf{V}=\left[\begin{array}{cc}
\frac{53}{64} & \frac{11}{64} \\
\frac{1}{4} & \frac{3}{4}
\end{array}\right], \\
\mathbf{c}=[-1,1]^{T}, \quad \mathbf{W}=\left[\mathbf{q}_{0}, \mathbf{q}_{1}, \mathbf{q}_{2}\right]=\left[\begin{array}{ccc}
1 & -1 & \frac{1}{2} \\
1 & 1 & \frac{1}{2}
\end{array}\right],
\end{gathered}
$$


while, for the values given by (3.10) the method has the coefficients:

$$
\begin{gathered}
\mathbf{B}=\left[\begin{array}{cc}
\frac{99}{128} & \frac{3}{128} \\
0 & \frac{5}{4}
\end{array}\right], \quad \mathbf{V}=\left[\begin{array}{cc}
\frac{243}{256} & \frac{13}{256} \\
\frac{1}{16} & \frac{15}{16}
\end{array}\right], \\
\mathbf{c}=[-2,2]^{T}, \quad \mathbf{W}=\left[\mathbf{q}_{0}, \mathbf{q}_{1}, \mathbf{q}_{2}\right]=\left[\begin{array}{ccc}
1 & -2 & 2 \\
1 & 2 & 2
\end{array}\right] .
\end{gathered}
$$

In order to reach higher values for $\operatorname{SSP}$ coefficient $\mathcal{C}$, in the following subsection we consider methods of stage order $q=1$.

\subsubsection{Methods with $p=r=s=2, q=1$}

Solving the order conditions for order $p=2$ and stage order $q=1$ we obtain a 7-parameter family depending on $c_{1}, c_{2}, v_{1}, v_{2}, q_{12}, q_{22}$ and $a_{21}$ under the restriction $\left|v_{1}-v_{2}\right|<1$. Since we are looking for SSP methods, Lemma 1 implies $a_{21}=0$. Maximizing the SSP coefficient $\mathcal{C}$, adding the restriction $-1 \leq c_{1}<c_{2} \leq 1$, we obtain:

$$
\mathcal{C}=\frac{3}{4} \text { for } c_{1}=-1, c_{2}=1, v_{1}=\frac{7}{10}, v_{2}=\frac{1}{10} \text { and } q_{12}=q_{22}+3,
$$

where $q_{22}$ is still a free parameter. If we consider the restriction $-2 \leq c_{1}<$ $c_{2} \leq 2$, the value for $\mathcal{C}$ increases to $\frac{7}{8}$. In this case we have

$$
\mathcal{C}=\frac{7}{8} \text { for } c_{1}=-2, c_{2}=2, v_{1}=\frac{29}{36}, v_{2}=\frac{1}{36} \text { and } q_{12}=q_{22}+10,
$$

where $q_{22}$ is still a free parameter. In this case, it seems that the maximal values of the coefficient $\mathcal{C}$ the following relation holds:

$$
\mathcal{C}=1-\frac{1}{2\left(c_{2}-c_{1}\right)}
$$

\subsection{Methods with $p=r=s=3$}

In this section we investigate methods with $p=r=s=3$ and $q=3,2,1$. In this and in the following section we assume that $c_{s}=1$. Relaxing this condition allows abscissas shifting, but seems not to lead to larger values for the SSP coefficient.

\subsubsection{Methods with $p=r=s=3, q=3$}

Forcing strictly increasing abscissas, and $c_{3}=1$, the optimal SSP method has $\mathcal{C}=\sqrt{2}-1$ and the following coefficients:

$$
\begin{gathered}
\mathbf{c}=\left[\begin{array}{lll}
-1-\sqrt{2} & -\sqrt{2} & 1
\end{array}\right]^{T}, \quad \mathbf{B}=\left[\begin{array}{ccc}
0 & 0 & 0 \\
0 & 4-2 \sqrt{2} & 0 \\
\frac{5}{2}-\frac{3}{\sqrt{2}} & 0 & \frac{9}{2}-2 \sqrt{2}
\end{array}\right], \\
\mathbf{V}=\left[\begin{array}{ccc}
0 & 1 & 0 \\
-1+\sqrt{2} & -8+6 \sqrt{2} & 10-7 \sqrt{2} \\
\frac{19}{2}-\frac{13}{\sqrt{2}} & 0 & \frac{13}{\sqrt{2}}-\frac{17}{2}
\end{array}\right] .
\end{gathered}
$$


For this method $\mathcal{C}_{\text {eff }}=\frac{\sqrt{2}-1}{3} \approx 0.138$.

\subsubsection{Methods with $p=r=s=3, q=2$}

In this case, forcing the restriction $-5 \leq c_{1}<c_{2}<c_{3}=1$, we obtain that the optimal SSP method has $\mathcal{C}=\frac{5}{6}$, while under the restriction $-3 \leq c_{1}<c_{2}<$ $c_{3}=1$, we obtain that the optimal SSP method has $\mathcal{C}=\frac{3}{4}$. The coefficients of this last method are:

$$
\begin{gathered}
\mathbf{B}=\left[\begin{array}{ccc}
\frac{1317487}{1769472} & \frac{7973}{10592} & \frac{1075}{589824} \\
\frac{4955}{36864} & \frac{2089}{2304} & \frac{2543}{12288} \\
0 & 0 & \frac{5}{4}
\end{array}\right], \quad \mathbf{V}=\left[\begin{array}{ccc}
\frac{3851}{4096} & \frac{473}{8192} & \frac{17}{8192} \\
\frac{35}{256} & \frac{359}{512} & \frac{83}{512} \\
\frac{1}{16} & 0 & \frac{15}{16}
\end{array}\right], \\
\mathbf{c}=\left[\begin{array}{lll}
-3 & 0 & 1
\end{array}\right]^{T}, \quad \mathbf{W}=\left[\mathbf{q}_{0}, \mathbf{q}_{1}, \mathbf{q}_{2}, \mathbf{q}_{3}\right]=\left[\begin{array}{cccc}
1 & -3 & \frac{9}{2} & 0 \\
1 & 0 & 0 & -\frac{26}{9} \\
1 & 1 & \frac{1}{2} & -\frac{26}{3}
\end{array}\right] .
\end{gathered}
$$

Let us remark that for this method the value of $\mathcal{C}$ can be obtained as $\mathcal{C}=v_{33} / b_{33}=3 / 4$ (compare $(3.6)$ ) and so $\mathcal{C}_{\text {eff }}=1 / 4$. In this case, our numerical search points out that the best attainable value for $\mathcal{C}$ seems to be

$$
\mathcal{C}=1-\frac{1}{c_{3}-c_{1}}
$$

3.2.3 Methods with $p=r=s=3, q=1$

In this case the higher $\mathrm{SSP}$ coefficient $\mathcal{C}$ is given by

$$
\mathcal{C}=\frac{v_{12}}{b_{12}}=-z \approx 0.782286,
$$

where $z \approx-0.782286$ is a root of the polynomial

$$
\begin{aligned}
p(x)= & -290965200+1284558760 x+2860364411 x^{2}-7981097947 x^{3}+ \\
& -19201804817 x^{4}-9167715999 x^{5}+1003129920 x^{6},
\end{aligned}
$$

and $v_{11}=1-v_{12} \approx 0.795767$ is a root of the polynomial

$$
\begin{aligned}
p(x)= & -120168990605590227+175319907436584132 x \\
& +41426367452248895 x^{2}-77002533780794860 x^{3} \\
& -16999999144346705 x^{4}+47246885203112 x^{5}+105800506695653 x^{6} .
\end{aligned}
$$

The coefficients of this methods are:

$$
\begin{gathered}
\mathbf{c}=\left[\begin{array}{ccc}
-3 & -\frac{29}{10} & 1
\end{array}\right]^{T}, \\
\mathbf{B}=\left[\begin{array}{ccc}
0.7185038451423001 & 0.26107280720903675 & 0 \\
0 & 0 & 0.2657061947964307 \\
0 & 0 & 1.2096037031663531
\end{array}\right], \\
\mathbf{V}=\left[\begin{array}{ccc}
0.7957665235133686 & 0.20423347648663137 & 0 \\
0.7635281794723168 & 0.028613712027478297 & 0.20785810850020528 \\
0 & 0.05374453927342392 & 0.9462554607265761
\end{array}\right],
\end{gathered}
$$

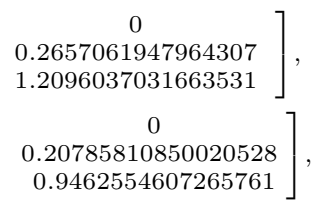


and

$$
\begin{aligned}
\mathbf{W} & =\left[\mathbf{q}_{0}, \mathbf{q}_{1}, \mathbf{q}_{2}, \mathbf{q}_{3}\right] \\
& =\left[\begin{array}{cccc}
1 & -3 & 3.8829228414636545 & 9.177166775938929 \\
1 & -2.9 & 5.903270746377843 & 0.4543609951704569 \\
1 & 1 & 0.5 & -10
\end{array}\right] .
\end{aligned}
$$

This method has $\mathcal{C}_{\text {eff }} \approx 0.261$. which represents just a slight improvement with respect to the value $\mathcal{C}_{\text {eff }}=1 / 4$ obtained for method (3.11)-(3.12) having same order $p=3$, but a higher stage order $q=2$.

\subsection{Methods with $p=r=s=4$}

In this section we investigate methods with $p=r=s=4$ and $q=1,2,3,4$.

\subsubsection{Methods with $p=r=s=4, q=1$}

Forcing strictly increasing abscissas, and $c_{4}=1$, the optimal SSP method has

\begin{tabular}{|c|c|c|c|c|}
\hline \multirow{4}{*}{$\mathbf{B}=$} & & & & \\
\hline & 0. & & & \\
\hline & $\begin{array}{l}105900001000000 \\
533513573132226\end{array}$ & 0.1316100010100260 & 0.2204010009040 & 3290 \\
\hline & 0 & 0 & 0 & 3180017841770 \\
\hline \multirow{4}{*}{$\mathbf{V}=$} & [0.5233434195205922 & 0.1482774935168031 & 0.3257753410935703 & 0.00260374586903437 \\
\hline & 0.0524219998842081 & 0.6508084969235142 & 0.1861196295058758 & 0.1106498736864019 \\
\hline & 0.1138926593620751 & 0.6605498625171727 & 0.2182774999048495 & 0.0072799782159028 \\
\hline & 0.2733912307975496 & 0.0000285832038595 & 0 & 0.7265801859985909 \\
\hline
\end{tabular}
$\mathcal{C} \approx 0.450$ and the following coefficients:

and

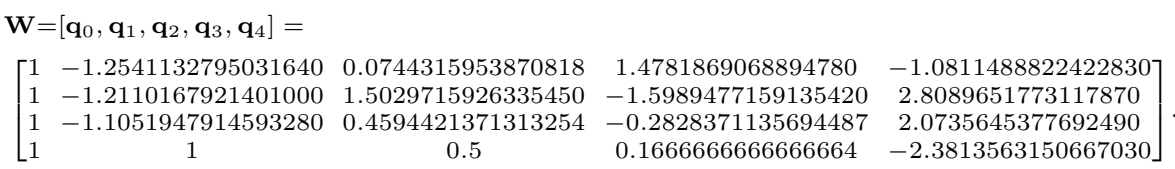

For this method $\mathcal{C}_{\text {eff }} \approx 0.112$.

\subsubsection{Methods with $p=r=s=4, q=2$}

In this case, forcing the restriction $-3 \leq c_{1}<c_{2}<c_{3}<c_{4}=1$, we obtain that the optimal SSP method has $\mathcal{C} \approx 0.507$, whose coefficients are

$\mathbf{c}=\left[\begin{array}{lccc}-3 & -1.5307929996603450 & -1.5207929996603450 & 1\end{array}\right]^{T}$,
$\mathbf{B}=\left[\begin{array}{cccc}0.3446530252761958 & 0 & 0 & 0.0026299108009584 \\ 0 & 0.5387417049219302 & 0.3156134630119571 & 0.1027295988157450 \\ 0 & 0.2900023222017333 & 1.0524245809100090 & 0.0430876450117072 \\ 0.3009115537533498 & 0 & 0 & 1.5509115537533500\end{array}\right]$,
$\mathbf{V}=\left[\begin{array}{cccc}0.5610128954739848 & 0 & 0.4376524983724827 & 0.0013346061535325 \\ 0.0647413008868319 & 0.2733963426787711 & 0.6097299591380859 & 0.0521323972963111 \\ 0.2968901298627457 & 0.1471680650188273 & 0.5340760307534204 & 0.0218657743650067 \\ 0.2129557768766749 & 0 & 0 & 0.7870442231233251\end{array}\right]$,


and

$$
\begin{aligned}
& \mathbf{W}=\left[\mathbf{q}_{0}, \mathbf{q}_{1}, \mathbf{q}_{2}, \mathbf{q}_{3}, \mathbf{q}_{4}\right]= \\
& {\left[\begin{array}{ccccc}
1 & -3 & 4.5 & -4.3842836159558560 & 4.5735139716803790 \\
1 & -1.5307929996603450 & 1.1716636039045580 & -0.3227609258267113 & 1.2832700522136430 \\
1 & -1.5207929996603450 & 1.1564056739079550 & -0.7092706562374267 & 2.2165808342746730 \\
1 & 1 & 0.5 & 0.1372706533030787 & -3.3681382547675550
\end{array}\right] .}
\end{aligned}
$$

For this method $\mathcal{C}_{\text {eff }} \approx 0.127$.

\subsubsection{Methods with $p=r=s=4, q=3$}

Forcing strictly increasing abscissas, and $c_{4}=1$, the optimal SSP method has $\mathcal{C} \approx 0.410$ and the following coefficients:

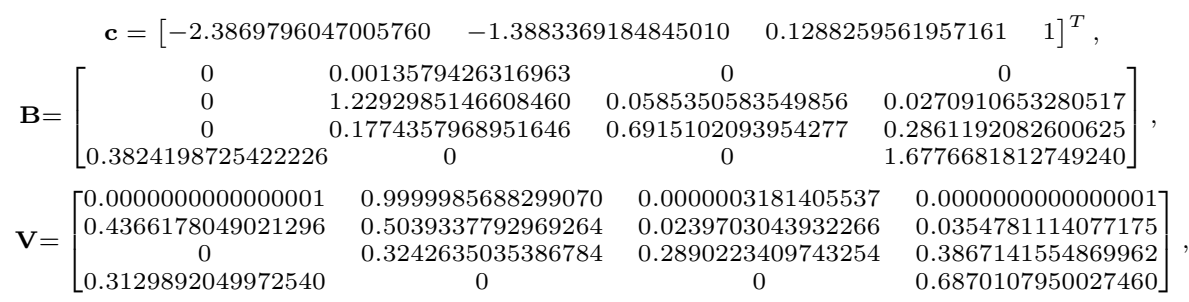

and

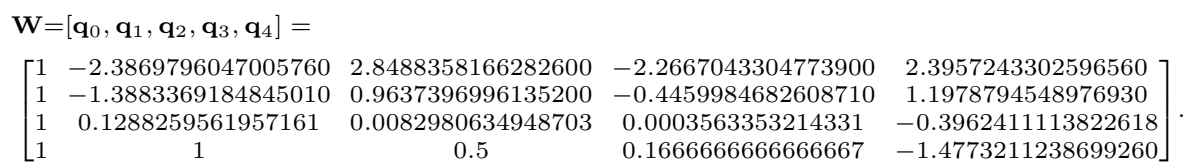

This method has $\mathcal{C}_{\text {eff }} \approx 0.102$.

\subsubsection{Methods with $p=r=s=4, q=4$}

After an extensive numerical search, we were not able to find SSP GLMs satisfying (3.1) with $p=q=r=s=4$.

The assumption $\mathbf{U}=\mathbf{I}$ in (3.1), allows only the construction of SSP GLMs having smaller SSP coefficients $\mathcal{C}$ than the corresponding SSP Runge-Kutta methods [23], two-step Runge-Kutta methods [24] and multistep multiderivative methods [8]. In the following section we will see that considering a more general coefficient matrix $\mathbf{U} \neq \mathbf{I}$ allows us to overcome this drawback, and to construct methods with quite large SSP coefficients.

\section{Methods with $p=r=s$ and $\mathbf{U} \neq \mathbf{I}$}

In this section we systematically investigate multistage integration methods of order $p=2,3$, and 4 and such that

$$
\mathbf{U} \neq \mathbf{I}, \quad \text { and } \quad p=r=s, \quad q=1,2, \ldots, p .
$$

As in Section 3.3, to compute the SSP coefficient $\mathcal{C}$ we numerically solved the minimization problem (2.5), by using the Matlab function fmincon and choosing 
Table 2. $\mathcal{C}_{\text {eff }}$ for GLMpqrs (2.1) with order $p=2$, stage order $q=1,2, r=2$ external stages and $s=2$ internal stages.

\begin{tabular}{cc}
\hline GLM2122 & GLM2222 \\
\hline 0.847 & 0.822 \\
\hline
\end{tabular}

Table 3. $\mathcal{C}_{\text {eff }}$ for GLMpqrs (2.1) with order $p=3$, stage order $q=1,2,3, r=3$ external stages and $s=3$ internal stages.

\begin{tabular}{ccc}
\hline GLM3133 & GLM3233 & GLM3333 \\
\hline 0.667 & 0.578 & 0.554 \\
\hline
\end{tabular}

Table 4. $\mathcal{C}_{\text {eff }}$ for GLMpqrs (2.1) with order $p=4$, stage order $q=1,2, \ldots, 4, r=4$ external stages and $s=4$ internal stages.

\begin{tabular}{cccc}
\hline GLM4144 & GLM4244 & GLM4344 & GLM4444 \\
\hline 0.532 & 0.529 & 0.518 & 0.504 \\
\hline
\end{tabular}

the sequential quadratic programming ('sqp') algorithm. Since fmincon is a local minimization routine, we ran an extensively numerical search using at least ten thousands random starting points, generated by means of the rand Matlab function. To the aim of speeding up this search for SSP methods when the number of free parameters and constraints increases the necessary condition provided by the following lemma can be useful (for example to provide sharper bounds for parameters and to obtain a better list of starting points).

Lemma 2. If a general linear method (2.1) has a positive SSP coefficient, $\mathcal{C}>0$, then

$$
0 \leq \mathbf{L e} \leq \mathbf{e}, \quad 0 \leq \gamma \mathbf{B L e} \leq \mathbf{e},
$$

where $\mathbf{L}=(\mathbf{I}+\gamma \mathbf{A})^{-1}$ and $0<\gamma \leq \mathcal{C}$.

Proof. Let us recall we are assuming that $\mathbf{U e}=\mathbf{e}$ and $\mathbf{V e}=\mathbf{e}$. From (2.3), $\mathbf{L U} \geq 0$ implies $\mathbf{L e}=\mathbf{L U e} \geq 0$, and $\mathbf{I}-\mathbf{L} \geq 0$ implies $\mathbf{L e} \leq \mathbf{I e}=\mathbf{e}$, hence the first part of inequalies (4.1) holds. Again, from (2.3), $\gamma \mathbf{B L} \geq 0$ implies $\gamma \mathbf{B L e} \geq 0$, and $\mathbf{V}-\gamma \mathbf{B L U} \geq 0$ implies $\gamma \mathbf{B L U e} \leq \mathbf{V e}$, that is $\gamma \mathbf{B L e} \leq \mathbf{e}$, and so the second part of inequalities (4.1) holds, too.

The $\mathcal{C}_{\text {eff }}$ coefficients for methods with two, three and four external stages are respectively listed in Tables 2, 3 and 4, using the notation GLMpqrs, where $p$ is the order of the method, $q$ is the stage order, $r$ is the number of external stages and $s$ is the number of internal stages.

Here we report the coefficient matrices of optimal SSP GLMs of order $p=2$, $p=3$ and $p=4$ and $p=q=r=s$. It is worth remarking that the coefficients listed here are computed using double precision accuracy and they are reported with 16 digits. A technique similar to the one used in [35] can be used when 
more accurate coefficients are needed, for example for implementation in an extended precision environment.

\section{Coefficients of GLM2222:}

$$
\begin{aligned}
& \mathbf{c}=\left[\begin{array}{ll}
0.5022655558767691 & 1
\end{array}\right]^{T}, \\
& \mathbf{A}=\left[\begin{array}{cc}
0 & 0 \\
0.5708860675842338 & 0
\end{array}\right], \quad \mathbf{U}=\left[\begin{array}{ll}
0.9184891352423395 & 0.0815108647576605 \\
0.8621853383442499 & 0.1378146616557501
\end{array}\right], \\
& \mathbf{B}=\left[\begin{array}{ll}
0.5708860675842338 & 0.6081669766552923 \\
0.2482943789611213 & 0.2645088930130668
\end{array}\right] \text {, } \\
& \mathbf{V}=\left[\begin{array}{ll}
0.8621853383442499 & 0.1378146616557501 \\
0.3749886103184382 & 0.6250113896815619
\end{array}\right] \text {, } \\
& \mathbf{W}=\left[\begin{array}{ccc}
1 & 0.6081669766552924 & -0.0000000000000001 \\
1 & -0.6910637589451494 & 1.5474666436813336
\end{array}\right] \text {. }
\end{aligned}
$$

This method has $\mathcal{C}_{\text {eff }} \approx 0.822$.

\section{Coefficients of GLM3333:}

$$
\begin{aligned}
& \mathbf{c}=\left[\begin{array}{lll}
0.3295839783544315 & 0.6806617112619909 & 1
\end{array}\right]^{T}, \\
& \mathbf{A}=\left[\begin{array}{ccc}
0 & 0 & 0 \\
0.5124026992885452 & 0 & 0 \\
0.4084203656103463 & 0.4796606306581744 & 0
\end{array}\right] \text {, } \\
& \mathbf{U}=\left[\begin{array}{ccc}
0 & 1 & 0 \\
0 & 0.8514777730453410 & 0.1485222269546588 \\
0.1313458703216458 & 0.6786866342802576 & 0.1899674953980965
\end{array}\right] \text {, } \\
& \mathbf{B}=\left[\begin{array}{ccc}
0.5223463949514766 & 0.5348295830910508 & 0 \\
0.3347759349512645 & 0.3931704919952592 & 0.4932702635381821 \\
0 & 0 & 0
\end{array}\right] \text {, } \\
& \mathbf{V}=\left[\begin{array}{ccc}
0 & 0.8680015654661640 & 0.1319984345338356 \\
0.2607207697861334 & 0.5563090669843533 & 0.1829701632295133 \\
1 & 0 & 0
\end{array}\right] \text {, } \\
& \mathbf{W}=\left[\begin{array}{cccc}
1 & 0.2433831470792890 & -0.1453586170258652 & 0.0319049749709932 \\
1 & 0.3295839783544315 & 0.0543127993939672 & 0.0059668761666100 \\
1 & -0.7566168529207110 & 0.1112582358948458 & 0.1322884988698362
\end{array}\right] \text {. }
\end{aligned}
$$

This method has $\mathcal{C}_{\text {eff }} \approx 0.554$.

\section{Coefficients of GLM4444:}

$\mathbf{c}=[0.2389332461541251$

$\mathbf{A}=\left[\begin{array}{c}0 \\ 0.3876590107850190 \\ 0.3052895098296686 \\ 0.2489878897536953\end{array}\right.$

$\mathbf{U}=\left[\begin{array}{l}0.0912599380251995 \\ 0.2893536053383063 \\ 0.3134061212806464 \\ 0.2556076322999006\end{array}\right.$

$\mathbf{B}=\left[\begin{array}{l}0.4084337666596042 \\ 0.3828018917763514 \\ 0.2475481687517574 \\ 0.1081881397565976\end{array}\right.$

$\mathbf{V}=\left[\begin{array}{l}0.0751122991274016 \\ 0.2875479264730055 \\ 0.2204520296708872 \\ 0.1110644950381738\end{array}\right.$
0.4860573286209339

0
0
0.3907983774045524
0.3187271759302078

\section{0}

0.1269451063377834 0.2876226025113369

$$
0
$$

0.4900212858838719

0.2657257201220867

0.1384906723289790

0.1721149858574717

0.0027007530079466

0.3255492887593254

0.2259735764529201 $\left.\begin{array}{ll}0.7359123877762289 & 1\end{array}\right]$,

$\left.\begin{array}{cc}0 & 0 \\ 0 & 0 \\ 0 & 0 \\ 0.4047222644510253 & 0\end{array}\right]$,

$\begin{array}{lll}0.9077024591751040 & 0.0010376027996967\end{array}$ $\begin{array}{lll}0.7090920916129786 & 0.0015543030487151\end{array}$ $\left.\begin{array}{lll}0.5584247264993190 & 0.0012240458822511\end{array}\right]$, $\left.\begin{array}{ll}0.4554398030738938 & 0.0013299621148688\end{array}\right]$

\section{0}

0.0105574651913504

0.3374206007279392

0.1758565404620620

0.7470925370715471

0.7002076220630648

0.4528063165607442

0.1978939020464726

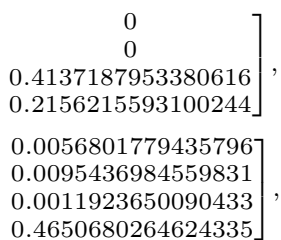


and

$$
\mathbf{W}=\left[\begin{array}{ccccc}
1 & -0.4024799023418847 & 0.0109880388418833 & 0.0335886630738861 & 0 \\
1 & -0.0255262786434428 & -0.1034566363144243 & 0.0182430084929503 & 0.0229216637127512 \\
1 & 0.3044460558739243 & 0.0291029904711275 & 0.0007065225928177 & -0.0019072286304098 \\
1 & -0.6581482865003410 & 1.0841569582135440 & -1.3812585734008830 & 1.7993346354617410
\end{array}\right]
$$

This method has $\mathcal{C}_{\text {eff }} \approx 0.504$.

\section{$5 \quad$ Starting and Finishing Procedures}

It is the worth remarking that the multistage integration methods considered in this paper require a starting vector $y^{[0]}$ which satisfies the relation $(2.2)$. In other words, these methods need a suitable starting procedure to attain the expected order of convergence $[2,3,39]$. In our implementation, we used starting procedures in the form of a generalized explicit RK method

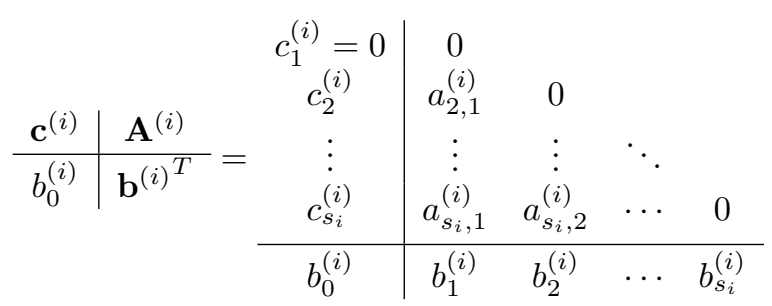

with $s_{i}$ stages, $i=1, \ldots, r$. Order conditions and a complete description of the construction of starting procedures of the form (5.1) can be found in [37].

Furthermore, from (2.2), the external stages of the GLMs (2.1) approximate a linear combination of the solution and its derivatives at grid points. So, usually, a finishing procedure is needed to recover an approximation for $y\left(t_{\text {end }}\right)$. This finishing procedure, which is a suitable linear combination of external stages, does not involve any additional evaluation of the function $f$ and can be constructed by using information coming only from the previous integration step. A possible way to determine a finishing procedure is the following. From (2.2) we know that

$$
y_{i}^{[n]}=\sum_{k=0}^{p} q_{i k} h^{k} y^{(k)}\left(t_{n}\right)+O\left(h^{p+1}\right), \quad i=1,2, \ldots, r,
$$

and so

$$
y_{i}^{[n-1]}=\sum_{k=0}^{p} q_{i k} h^{k} y^{(k)}\left(t_{n-1}\right)+O\left(h^{p+1}\right), \quad i=1,2, \ldots, r .
$$

Expanding $y^{(k)}\left(t_{n-1}\right)$ in Taylor series about $t_{n}$, up to the power $p-k$, we obtain

$$
y^{(k)}\left(t_{n-1}\right)=\sum_{j=0}^{p-k} \frac{(-1)^{j} h^{j}}{j !} y^{(k+j)}\left(t_{n}\right)+O\left(h^{p-k+1}\right),
$$


and hence, for $i=1,2, \ldots, r$, we have

$$
\begin{aligned}
y_{i}^{[n-1]} & =\sum_{k=0}^{p} q_{i k} h^{k}\left(\sum_{j=0}^{p-k} \frac{(-1)^{j} h^{j}}{j !} y^{(k+j)}\left(t_{n}\right)+O\left(h^{p-k+1}\right)\right)+O\left(h^{p+1}\right) \\
& =\sum_{k=0}^{p} q_{i k}\left(\sum_{j=0}^{p-k} \frac{(-1)^{j} h^{k+j}}{j !} y^{(k+j)}\left(t_{n}\right)\right)+O\left(h^{p+1}\right) \\
& =\sum_{k=0}^{p} \sum_{m=k}^{p} q_{i k} \frac{(-1)^{m-k} h^{m}}{(m-k) !} y^{(m)}\left(t_{n}\right)+O\left(h^{p+1}\right) \\
& =\sum_{m=0}^{p} \sum_{k=0}^{m} q_{i k} \frac{(-1)^{m-k} h^{m}}{(m-k) !} y^{(m)}\left(t_{n}\right)+O\left(h^{p+1}\right) \\
& =\sum_{m=0}^{p}\left(\sum_{k=0}^{m} \frac{(-1)^{m-k} q_{i k}}{(m-k) !}\right) h^{m} y^{(m)}\left(t_{n}\right)+O\left(h^{p+1}\right) .
\end{aligned}
$$

So we obtain

$$
y_{i}^{[n-1]}=\sum_{k=0}^{p}\left(\sum_{m=0}^{k} \frac{(-1)^{k-m} q_{i m}}{(k-m) !}\right) h^{k} y^{(k)}\left(t_{n}\right)+O\left(h^{p+1}\right), i=1,2, \ldots, r .
$$

Let us now consider the case $r=p$. If we define the matrix $\mathbf{W} \in \mathbb{R}^{r \times(p+1)}$ as $\mathbf{W}=\left[\mathbf{q}_{0}, \mathbf{q}_{1}, \ldots, \mathbf{q}_{p}\right]$, we can recover a numerical approximation of order $p$ for the value $y\left(t_{n}\right)$ by considering a suitable linear system whose coefficient matrix can be obtained adding a row to the matrix $\mathbf{W}$. To this aim, let us define the row vector $\widetilde{\mathbf{q}} \in \mathbb{R}^{1 \times(p+1)}$ as

$$
\widetilde{\mathbf{q}}=\left[\sum_{m=0}^{k} \frac{(-1)^{k-m} q_{1 m}}{(k-m) !}\right]_{k=0, \ldots, p}
$$

We can consider the equality

$$
\widetilde{y}^{[n]}=\mathbf{Q} \widetilde{y}_{n}+O\left(h^{p+1}\right), \quad \mathbf{Q}=\left[\begin{array}{c}
\mathbf{W} \\
\widetilde{\mathbf{q}}
\end{array}\right] \in \mathbb{R}^{(p+1) \times(p+1)},
$$

where

$$
\widetilde{y}^{[n]}=\left[y_{1}^{[n]}, y_{2}^{[n]}, \ldots, y_{r}^{[n]}, y_{1}^{[n-1]}\right]^{T}, \quad \widetilde{y}_{n}=\left[y\left(t_{n}\right), y^{\prime}\left(t_{n}\right), \ldots, y^{(p)}\left(t_{n}\right)\right]^{T} .
$$

When the matrix $\mathbf{Q}$ is invertible, then $\widetilde{y}_{n}=\mathbf{Q}^{-1} \widetilde{y}^{[n]}+O\left(h^{p+1}\right)$, and hence an approximation for the value of $y\left(t_{n}\right)$ can be computed as a scalar product of the first row of $\mathbf{Q}^{-1}$ by the vector $\widetilde{y}^{[n]}$. If the matrix $\mathbf{Q}$ is not invertible, as in case of GLM3333, then the linear system can be obtained by choosing in a different way the $p+1$ equations between the $2 r$ equations given by (2.1) and (5.3). 


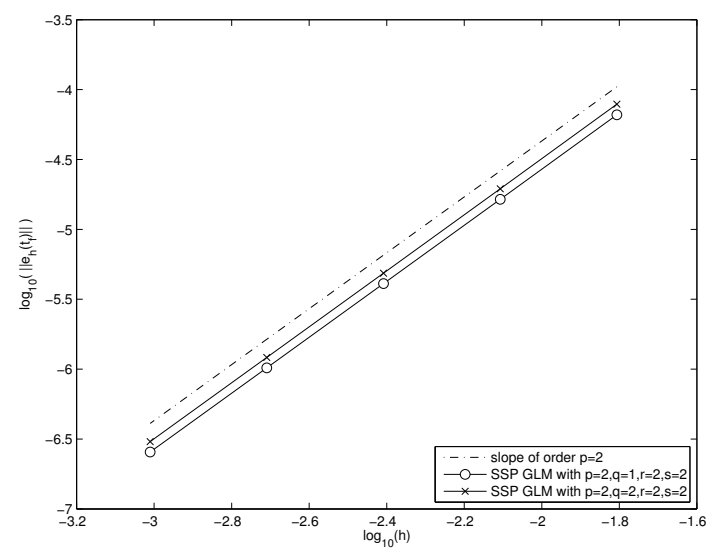

Figure 1. Order verification for SSP GLMs of order $p=2$ and stage order $q=1$ and 2 .

\section{$6 \quad$ Numerical Experiments}

\subsection{Validation of convergence}

To verify the order of convergence we apply the methods derived in this paper to the system of ODEs obtained by first order upwind discretization in space variable $x$ of the test problem from $[8,49]$

$$
\frac{\partial y(x, t)}{\partial t}=-\frac{\partial y(x, t)}{\partial x}+\frac{t-x}{(1+t)^{2}}, \quad 0 \leq x \leq 1,0 \leq t \leq 1
$$

with initial condition $y(x, 0)=1+x, 0 \leq x \leq 1$, and left boundary condition $y(0, t)=1 /(1+t), 0 \leq t \leq 1$. The exact solution to this problem is

$$
y(x, t)=\frac{1+x}{1+t}, \quad 0 \leq x \leq 1,0 \leq t \leq 1 .
$$

Since this solution is linear in space variable $x$, the resulting system of ODEs does not introduce any discretization error and have the solution

$$
y_{i}(t)=y\left(x_{i}, t\right), \quad 0 \leq t \leq 1,
$$

for any grid point $x_{i}, i=0, \ldots, N$, where $x_{i}=i \Delta x, i=0,1, \ldots, N$, and $N \Delta x=1$.

We have plotted on Figs. 1-3 in double logarithmic scale the norm of global error at the end point $t_{f}$ of the interval of integration versus the temporal stepsize $h$ for methods of order $p=2, p=3, p=4$, and stage order $q=1,2, \ldots, p$. We can see that all methods achieve the expected order of convergence.

As already mentioned in the introduction the class of GLMs allows us to break the order four barrier for SSPRK methods. The work on the construction of SSP GLMs of high order and stage order will be reported elsewhere. 


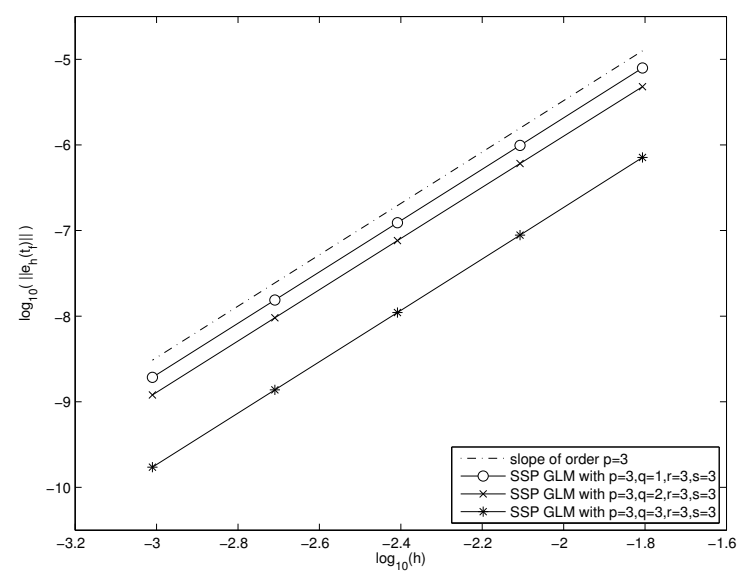

Figure 2. Order verification for GLMs of order $p=3$ and stage order $q=1,2$ and 3 .

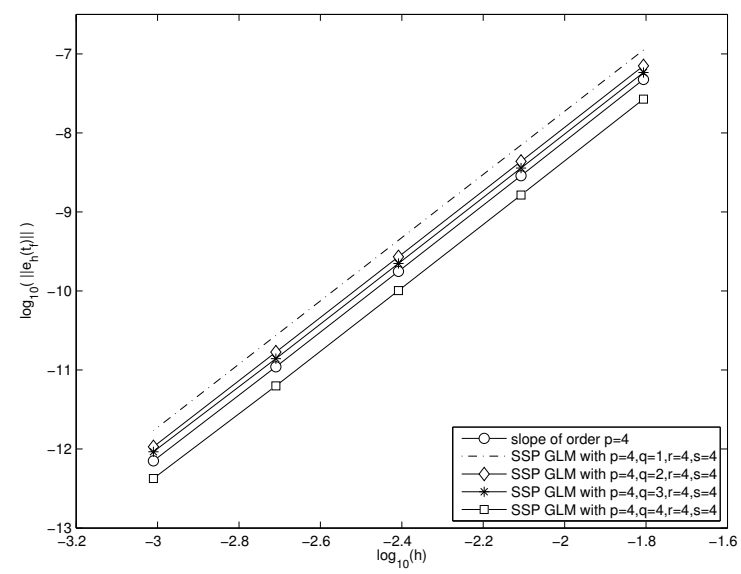

Figure 3. Order verification for GLMs of order $p=4$ and stage order $q=1,2,3$ and 4 .

\subsection{Order preservation for high stage order methods}

In order to further validate the order preservation for high stage order SSP multistage integration methods we report in Figs. 4 and 5 the results of numerical tests that point out that the constructed high order stages SSP multistage integration methods preserve the theoretical order of convergence $p$, while low stage order SSP Runge-Kutta methods suffer from the well known order reduction phenomenon. Specifically, following Constantinescu and Sandu [8], we considered problem (6.1) and pointed out that, when the spatial and temporal grids are refined simultaneously, $\operatorname{SSPRK}(3,3)$ method $[22]$ and $\operatorname{SSPRK}(5,4)$ method $[24,44,53]$ only achieve order $p=2$, while GLM3333, of order $p=3$ and stage order $q=3$, preserves the expected order $p=3$ (see Fig. 5). 

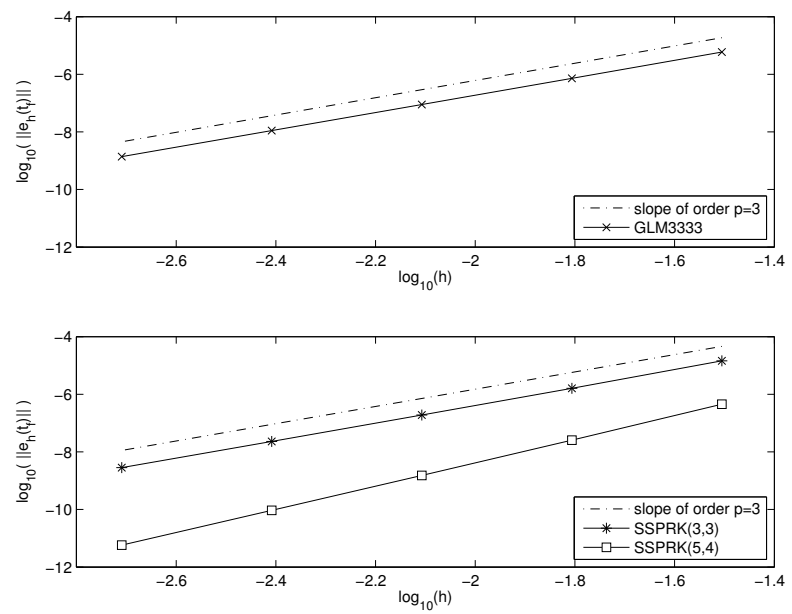

Figure 4. Order preservation for GLM3333, and for $\operatorname{SSPRK}(3,3)$ and $\operatorname{SSPRK}(5,4)$ on problem (6.1) when only the temporal grid is refined.
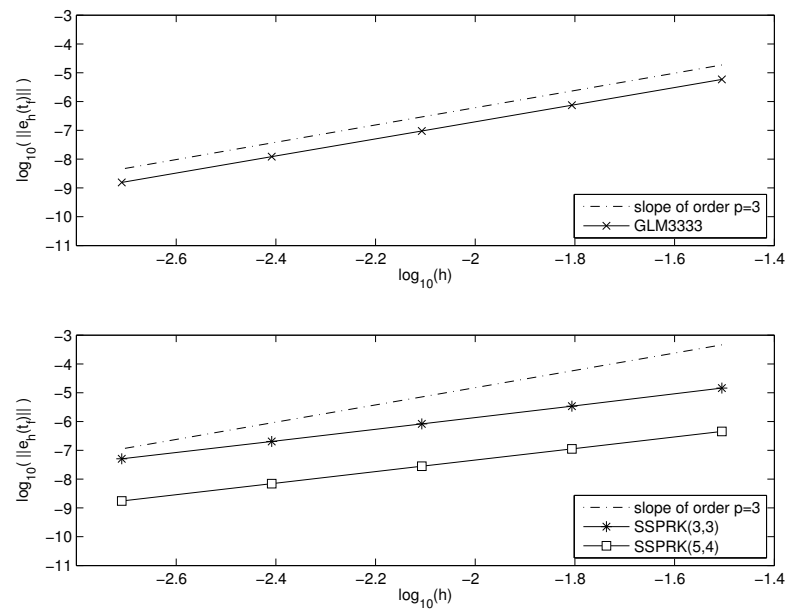

Figure 5. Order preservation for GLM3333 (top figure) and order reduction phenomenon for $\operatorname{SSPRK}(3,3)$ and $\operatorname{SSPRK}(5,4)$ (bottom figure) on problem (6.1) when spatial and temporal grids are refined simultaneously.

For the sake of completeness, it is worth to remark that when the space grid is maintained fixed, i.e. the ODE problem is fixed, then the expected order is preserved for all considered Runge-Kutta and multistage integration methods (see Fig. 4). The order reduction phenomenon for RK methods reported in Fig. 5 is due to naive implementation of a time-dependent Dirichlet boundary condition. This phenomenon has been analyzed in the literature and for linear hyperbolic equations it can be reduced or avoided by a suitable transformation or differentiation of the boundary conditions as shown in $[7,34,48,49]$. 


\subsection{Validation of monotonicity}

To verify monotonicity properties of GLMs constructed in this paper we consider, following Ferracina and Spijker [21], Constantinescu and Sandu [8] and Ketcheson et. al. [42,43], the following problems (compare also with $[34,45,53]$ ).

Problem 1. The inviscid Burgers equation

$$
\frac{\partial y(x, t)}{\partial t}+\frac{\partial}{\partial x}\left(\frac{1}{2} y^{2}(x, t)\right)=0, \quad 0 \leq x \leq X, 0 \leq t \leq t_{f},
$$

with $X=2$, and with initial condition

$$
y(x, 0)=\frac{1}{2}-\frac{1}{4} \sin (\pi x)
$$

and periodic boundary conditions $y(0, t)=y(2, t), 0 \leq t \leq t_{f}$.

Problem 2. We consider again the Burgers equation (6.2). In this case with $X=1$ and with discontinuous initial condition

$$
y(x, 0)= \begin{cases}1, & 0.18 \leq x \leq 0.44, \\ 0, & \text { otherwise }\end{cases}
$$

and periodic boundary conditions $y(0, t)=y(1, t), 0 \leq t \leq t_{f}$.

Problem 3. The Buckley-Leverett equation

$$
\frac{\partial y(x, t)}{\partial t}+\frac{\partial}{\partial x}(\Phi(y(x, t)))=0, \quad-1 \leq x \leq 1,0 \leq t \leq t_{f},
$$

where

$$
\Phi(y)=\frac{y^{2}}{y^{2}+a(1-y)^{2}} .
$$

This equation models a two-phase flow through the porous media, see for example [46]. We take $a=1 / 4$ and assume the discontinuous initial condition

$$
y(x, 0)= \begin{cases}1, & -0.5 \leq x \leq 0 \\ 0, & \text { otherwise }\end{cases}
$$

and periodic boundary conditions

$$
y(-1, t)=y(1, t), \quad 0 \leq t \leq t_{f} .
$$

For all these problems the space derivative was discretized by a fifth order finite difference weighted essentially non-oscillatory (WENO) scheme [51].

A numerical approximation obtained by the SSP multistage integration methods of order $p=3$ and stage order $q=3$ whose coefficients are listed in Section 4, is presented in Fig. 6, Fig. 7 and Fig. 8. For comparison purpose we have also also plotted the numerical approximation obtained by the $\operatorname{SSPRK}(3,3)$ method [22]. We can see that, using the same temporal stepsize 


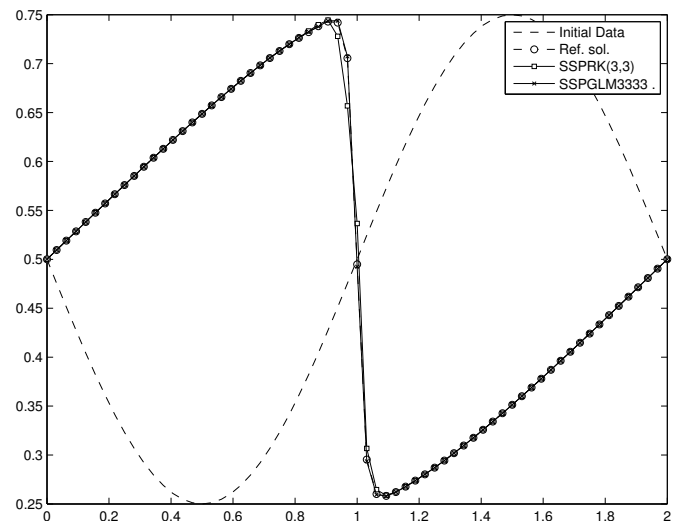

Figure 6. Numerical approximations at $t_{f}=2$ to the discretization of Problem 1, with $N=64, N_{t}=44$ and $\nu=1.091$, obtained by SSP GLM of order $p=3$ and stage order $q=3$, and by $\operatorname{SSPRK}(3,3)$.

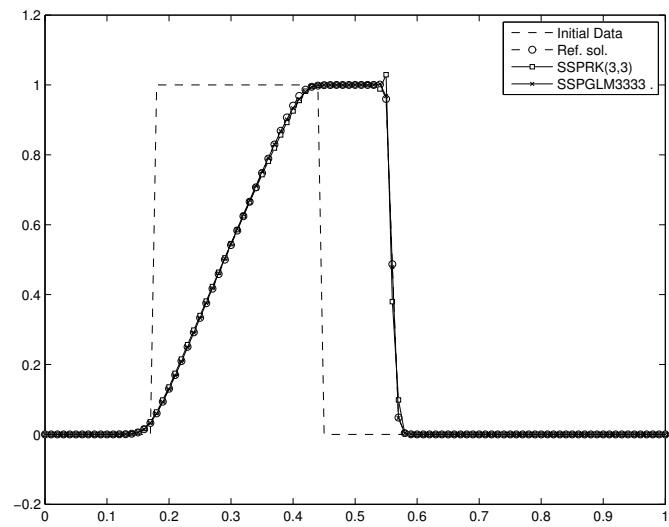

Figure 7. Numerical approximations at $t_{f}=0.23$ to the discretization of Problem 2, with $N=100, N_{t}=19$ and $\nu=1.211$,obtained by SSP GLM of order $p=3$ and stage order $q=3$, and by $\operatorname{SSPRK}(3,3)$.

$\Delta t$ for both methods, the SSP multistage integration methods exhibits smooth behavior, while numerical solution obtained by $\operatorname{SSPRK}(3,3)$ method exhibits spurious oscillations.

In the caption of Figures $6-8$ we denoted with $\nu$ the so-called Courant number (or CFL number) defined as $\nu=|a \Delta t / \Delta x|$, where $a=\max (y)$ for Problems 1 and 2 , and $a=\max \left(\frac{d \Phi}{d y}(y)\right)$ for Problem $3, \Delta t=t_{f} / N_{t}$ and $\Delta x=X / N$.

As is well known, the solution of hyperbolic PDEs can develop discontinuities, even if the initial data is a smooth function. In Problem 1 a shock occurs at $t=4 / \pi$ as the characteristic lines cross. For Problems 2 and 3 the presence of shocks is due to the discontinuity in the initial data. Our numerical results confirm that the presented SSP multistage integration methods methods, combined with appropriate spatial discretization, perform well even if a shock 


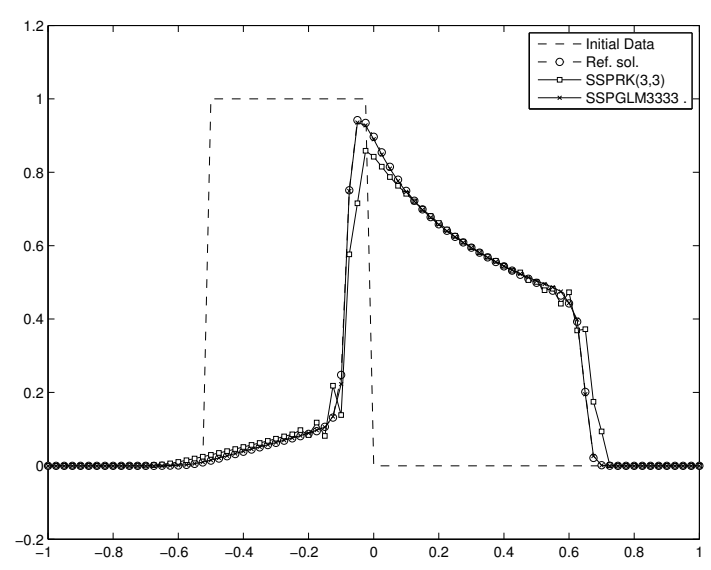

Figure 8. Numerical approximations at $t_{f}=0.4$ to the discretization of Problem 3 with $N=80, N_{t}=28$ and $\nu=1.493$, obtained by SSP of order $p=3$ and stage order $q=3$, and by $\operatorname{SSPRK}(3,3)$.

occurs.

Even if, for the sake of brevity, we are not reporting the results here, we verified that for sufficiently small values of CFL number $\nu$ the behavior of the methods derived in this paper is very similar to a SSPRK of the corresponding order (we used $\operatorname{SSP}(2,2), \operatorname{SSPRK}(3,3)$ and $\operatorname{SSPRK}(5,4)$ from [22]) for several space discretization schemes (such as first, second and third order upwind, second order ENO, Lax-Wendroff, third-order upwind-biased flux limited [34, 45]).

Finally we report the results of the investigation of the SSP property by means of the total variation (TV) semi-norm:

$$
T V(y(t, x))=\sum_{i=1}^{N}\left|y\left(t, x_{i}\right)-y\left(t, x_{i-1}\right)\right|,
$$

(compare [8]). The preservation of the strong stability requires that the TV norm be nonincreasing from one step to the next. It follows that the maximum total variation change is nonpositive

$$
\max _{i=1, \ldots, N_{t}}\left(T V\left(y\left(t_{i}, x\right)\right)-T V\left(y\left(t_{i-1}, x\right)\right)\right) \leq 0, \quad N_{t}=t_{f} / \Delta t .
$$

In Figure 9 we plotted the maximum TV change for the numerical solution obtained by applying the Forward Euler method and the multistage integration methods listed in Section 4 to Problem 1 using the conservative first order upwind scheme for the spatial discretization, with $N=100$ and $t_{f}=2$. For comparison, we also reported in Figure 10 an analogous plot obtained by the Runge-Kutta methods $\operatorname{SSP}(2,2), \operatorname{SSPRK}(3,3)$ and $\operatorname{SSPRK}(5,4)$ from $[22]$. Figures 9 and 10 show that, in this case, GLM2222 and GLM3333 preserve the monotonicity of the numerical solution for larger Courant numbers (and hence for larger temporal stepsize) than $\operatorname{SSPRK}(2,2)$ and $\operatorname{SSPRK}(3,3)$, respectively. 


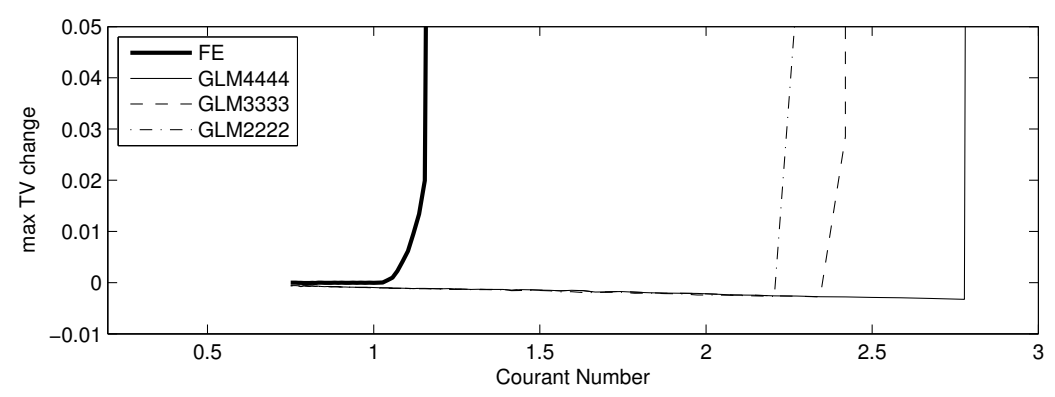

Figure 9. Maximum change of the Total Variation vs Courant Number, for the numerical solution to Problem 1, with $N=100$ and $t_{f}=2$, obtained by Forward Euler, GL2222, GL3333 and GL4444.

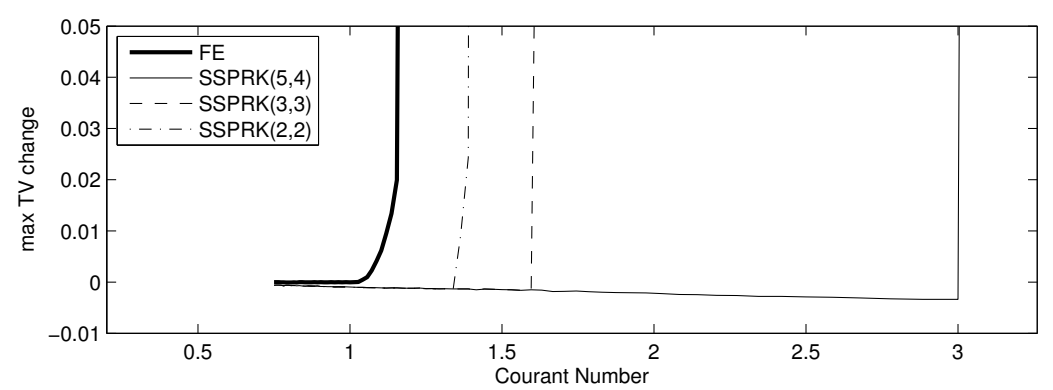

Figure 10. Maximum change of the Total Variation vs Courant Number, for the numerical solution to Problem 1 , with $N=100$ and $t_{f}=2$, obtained by Forward Euler, $\operatorname{SSPRK}(2,2), \operatorname{SSPRK}(3,3)$ and $\operatorname{SSPRK}(5,4)$.

A similar result holds for GLM4444 respect to $\operatorname{SSPRK}(5,4)$, if one take into account that $\operatorname{SSPRK}(5,4)$ has order four, but it has five stages, so the Courant number should be scaled by a factor of $4 / 5$. Hence $\operatorname{SSPRK}(5,4)$ would preserve the monotonicity of the numerical solution approximately up to an effective Courant number of 2.4 (compare [53]).

\section{Concluding Remarks}

We have used the monotonicity theory of general linear methods developed by Spijker [52] to construct methods which preserve the so-called strong stability property. To this aim, we systematically investigated the class of GLMs of order $p=2,3,4$, stage order $q=1,2, \ldots, p$ and such that the number of external stages $r$ and the number of internal stages $s$ both match the order of the method $p$. We also gave a characterization of methods for which $\mathbf{U}=\mathbf{I}$, and some useful necessary conditions to limit the search domain for the minimization problem (2.5) which is described in Section 2.3.

Numerical examples illustrate that the methods derived in this paper achieve the expected order of accuracy, and high stage order methods do not suffer 
from the order reduction phenomenon. Moreover, under appropriate stepsize restrictions, these methods combined with appropriate spatial discretization, do not produce spurious oscillations when applied to semidiscretizations of hyperbolic conservation laws.

Future work will address the construction of implicit SSP GLMs and explicit SSP GLMs of high order, and the efficient implementation of these methods for semidiscretizations in space variables of partial differential equations.

\section{Acknowledgments}

The results reported in this paper were obtained during the visit of the first author (GI) to the Arizona State University in the Spring semester of 2014. This author wish to express his gratitude to the School of Mathematical \& Statistical Sciences for hospitality during this visit.

\section{References}

[1] K. Burrage and J.C. Butcher. Non-linear stability of a general class of differential equation methods. BIT, 20(2):185-203, 1980.

http://dx.doi.org/10.1007/BF01933191.

[2] J.C. Butcher. Numerical Methods for Ordinary Differential Equations. Wiley, New York, 2003.

[3] J.C. Butcher. General linear methods. Acta Numer., 15:157-256, 2006. http://dx.doi.org/10.1017/S0962492906220014.

[4] A. Cardone, D. Conte and B. Paternoster. A family of multistep collocation methods for Volterra integro-differential equations. AIP Conf. Proc., 1168:358361, 2009. http://dx.doi.org/10.1063/1.3241469.

[5] A. Cardone and Z. Jackiewicz. Explicit Nordsieck methods with quadratic stability. Numer Algorithms, 60(1):1-25, 2012. http://dx.doi.org/10.1007/s11075-011-9509-y.

[6] A. Cardone, Z. Jackiewicz, J.H. Verner and B. Welfert. Order conditions for general linear methods. J. Comput. Appl. Math., 290:44-64, 2015. http://dx.doi.org/10.1016/j.cam.2015.04.042.

[7] M.H. Carpenter, D. Gottlieb, S. Abarbanel and W.-S. Don. The theoretical accuracy of Runge-Kutta time discretizations for the initial boundary value problem: A study of the boundary error. SIAM J. Sci. Comput., 16(6):1241-1252, 1995. http://dx.doi.org/10.1137/0916072.

[8] E.M. Constantinescu and A. Sandu. Optimal explicit strong-stability-preserving general linear methods. SIAM J. Sci. Comput., 32(5):3130-3150, 2010. http://dx.doi.org/10.1137/090766206.

[9] D. Conte, R. D'Ambrosio, Z. Jackiewicz and B. Paternoster. A practical approach for the derivation of algebraically stable two-step Runge-Kutta methods. Math. Model. Anal., 17(1):65-77, 2012. http://dx.doi.org/10.3846/13926292.2012.644870.

[10] D. Conte, R. D'Ambrosio and B. Paternoster. Advances on collocation based numerical methods for ordinary differential equations and Volterra integral equations. In Recent Advances in Computational and Applied Mathematics, pp. 41-66, 2011. 
[11] D. Conte, Z. Jackiewicz and B. Paternoster. Two-step almost collocation methods for Volterra integral equations. Appl. Math. Comput., 204(2):839-853, 2008. http://dx.doi.org/10.1016/j.amc.2008.07.026.

[12] R. D'Ambrosio, E. Esposito and B. Paternoster. General linear methods for $y "=f(y(t))$. Numer. Algorithms, 61(2):331-349, 2012. http://dx.doi.org/10.1007/s11075-012-9637-z.

[13] R. D'Ambrosio, G. Izzo and Z. Jackiewicz. Highly stable general linear methods for differential systems. AIP Conf. Proc., 1168:21-24, 2009. http://dx.doi.org/10.1063/1.3241431.

[14] R. D'Ambrosio, G. Izzo and Z. Jackiewicz. Perturbed MEBDF methods. Comput. Math. Appl., 63(4):851-861, 2012. http://dx.doi.org/10.1016/j.camwa.2011.11.050.

[15] R. D'Ambrosio, G. Izzo and Z. Jackiewicz. Search for highly stable two-step Runge-Kutta methods. Appl. Numer. Math., 62(10):1361-1379, 2012. http://dx.doi.org/10.1016/j.apnum.2012.06.012.

[16] R. D'Ambrosio, G. De Martino and B. Paternoster. Order conditions for general linear Nyström methods. Numer. Algorithms, 65(3):579-595, 2014. http://dx.doi.org/10.1007/s11075-013-9819-3.

[17] R. D'Ambrosio and B. Paternoster. A general framework for the numerical solution of second order ODEs. Math. Comput. Simulation, 110:113-124, 2015. http://dx.doi.org/10.1016/j.matcom.2014.04.007.

[18] L. Ferracina and M.N. Spijker. Stepsize restrictions for the total-variationdiminishing property in general Runge-Kutta methods. SIAM J. Numer. Anal., 42(3):1073-1093, 2004. http://dx.doi.org/10.1137/S0036142902415584.

[19] L. Ferracina and M.N. Spijker. An extension and analysis of the Shu-Osher representation of Runge-Kutta methods. Math. Comput., 74(249):201-219, 2005. http://dx.doi.org/10.1090/S0025-5718-04-01664-3.

[20] L. Ferracina and M.N. Spijker. Stepsize restrictions for total-variationboundedness in general Runge-Kutta procedures. Appl. Numer. Math., 53(24):265-279, 2005. http://dx.doi.org/10.1016/j.apnum.2004.08.024.

[21] L. Ferracina and M.N. Spijker. Strong stability of singly-diagonally-implicit Runge-Kutta methods. Appl. Numer. Math., 58(11):1675-1686, 2008. http://dx.doi.org/10.1016/j.apnum.2007.10.004.

[22] S. Gottlieb. On high order strong stability preserving Runge-Kutta and multi step time discretizations. J. Sci. Comput., 25(1):105-128, 2005. http://dx.doi.org/10.1007/s10915-004-4635-5.

[23] S. Gottlieb, D.I. Ketcheson and C.-W. Shu. High order strong stability preserving time discretizations. J. Sci. Comput., 38(3):251-289, 2009. http://dx.doi.org/10.1007/s10915-008-9239-z.

[24] S. Gottlieb, D.I. Ketcheson and C.-W. Shu. Strong Stability Preserving RungeKutta and Multistep Time Discretizations. World Scientific, 2011. ISBN 9789814289269. http://dx.doi.org/10.1142/7498.

[25] S. Gottlieb and S.J. Ruuth. Optimal strong-stability-preserving time-stepping schemes with fast downwind spatial discretizations. J. Sci. Comput., 27(13):289-303, 2006. http://dx.doi.org/10.1007/s10915-005-9054-8.

[26] S. Gottlieb and C.-W. Shu. Total variation diminishing Runge-Kutta schemes. Math. Comput., 67(221):73-85, 1998.

http://dx.doi.org/10.1090/S0025-5718-98-00913-2. 
[27] S. Gottlieb, C.-W. Shu and E. Tadmor. Strong stability-preserving high-order time discretization methods. SIAM Rev., 43(1):89-112, 2001.

[28] I. Higueras. On strong stability preserving time discretization methods. J. Sci. Comput., 21(2):193-223, 2004. http://dx.doi.org/10.1023/B:JOMP.0000030075.59237.61.

[29] I. Higueras. Monotonicity for Runge-Kutta methods: Inner product norms. $J$. Sci. Comput., 24(1):665-685, 2005. http://dx.doi.org/10.1007/s10915-004-4789-1.

[30] I. Higueras. Representations of Runge-Kutta methods and strong stability preserving methods. SIAM J. Numer. Anal., 43(3):924-948, 2005. http://dx.doi.org/10.1137/S0036142903427068.

[31] I. Higueras. Strong stability for Runge-Kutta schemes on a class of nonlinear problems. J. Sci. Comput., 57(3):518-535, 2013. http://dx.doi.org/10.1007/s10915-013-9715-y.

[32] W. Hundsdorfer and S.J. Ruuth. On monotonicity and boundedness properties of linear multistep methods. Math. Comput., 75(254):655-672, 2006. http://dx.doi.org/10.1090/S0025-5718-05-01794-1.

[33] W. Hundsdorfer, S.J. Ruuth and R.J. Spiteri. Monotonicity-preserving linear multistep methods. SIAM J. Numer. Anal., 41(2):605-623, 2003. http://dx.doi.org/10.1137/S0036142902406326.

[34] W.H. Hundsdorfer and J.G. Verwer. Numerical Solution of Time-Dependent Advection-Diffusion-Reaction Equations. Springer Ser. Comput. Math. Springer, Berlin, Heidelberg, New York, 2003.

[35] G. Izzo and Z. Jackiewicz. Construction of algebraically stable DIMSIMs. J. Comput. Appl. Math., 261:72-84, 2014.

http://dx.doi.org/10.1016/j.cam.2013.10.037.

[36] G. Izzo and Z. Jackiewicz. Construction of strong stability preserving general linear methods. AIP Conf. Proc., 1648, 2015. http://dx.doi.org/10.1063/1.4912441.

[37] G. Izzo and Z. Jackiewicz. Strong stability preserving general linear methods. J. Sci. Comput., 2015. http://dx.doi.org/10.1007/s10915-014-9961-7. (in press)

[38] G. Izzo, Z. Jackiewicz, E. Messina and A. Vecchio. General linear methods for Volterra integral equations. J. Comput. Appl. Math., 234(9):2768-2782, 2010.

[39] Z. Jackiewicz. General Linear Methods for Ordinary Differential Equations. John Wiley, Hoboken, New Jersey, 2009. ISBN 9780470408551. http://dx.doi.org/10.1002/9780470522165.

[40] Z. Jackiewicz and S. Tracogna. A general class of two-step Runge-Kutta methods for ordinary differential equations. SIAM J. Numer. Anal., 32(5):1390-1427, 1995. http://dx.doi.org/10.1137/0732064.

[41] Z. Jackiewicz and R. Vermiglio. General linear methods with external stages of different orders. BIT Numer. Math., 36(4):688-712, 1996. http://dx.doi.org/10.1007/BF01733788.

[42] D.I. Ketcheson, S. Gottlieb and C.B. Macdonald. Strong stability preserving two-step Runge-Kutta methods. SIAM J. Numer. Anal., 49(6):2618-2639, 2011. http://dx.doi.org/10.1137/10080960X. 
[43] D.I. Ketcheson, C.B. Macdonald and S. Gottlieb. Optimal implicit strong stability preserving Runge-Kutta methods. Appl. Numer. Math., 59(2):373-392, 2009. http://dx.doi.org/10.1016/j.apnum.2008.03.034.

[44] J.F.B.M. Kraaijevanger. Contractivity of Runge-Kutta methods. BIT, 31(3):482-528, 1991. http://dx.doi.org/10.1007/BF01933264.

[45] C.B. Laney. Computational Gasdynamics. Cambridge University Press, Cambridge, New York, NY, 1998.

[46] R.J. LeVeque. Finite Volume Methods for Hyperbolic Problems. Cambridge Texts Appl. Math. Cambridge University Press, Cambridge, New York, 2002.

[47] S.J. Ruuth and R.J. Spiteri. Two barriers on strong-stability-preserving time discretization methods. J. Sci. Comput., 17(1-4):211-220, 2002. http://dx.doi.org/10.1023/A:1015156832269.

[48] J.M. Sanz-Serna and J.G. Verwer. Convergence analysis of one-step schemes in the method of lines. Appl. Math. Comput., 31(C):183-196, 1989. http://dx.doi.org/10.1016/0096-3003(89)90118-5.

[49] J.M. Sanz-Serna, J.G. Verwer and W.H. Hundsdorfer. Convergence and order reduction of Runge-Kutta schemes applied to evolutionary problems in partial differential equations. Numer. Math., 50(4):405-418, 1987. http://dx.doi.org/10.1007/BF01396661.

[50] C.-W. Shu and S. Osher. Efficient implementation of essentially non-oscillatory shock-capturing schemes. J. Comput. Phys., 77(2):439-471, 1988. http://dx.doi.org/10.1016/0021-9991(88)90177-5.

[51] C.W. Shu. High order ENO and WENO schemes for computational fluid dynamics. In H. Deconinck(Ed.), High-Order Methods for Computational Physics, Lect. Notes Comput. Sci. Eng., pp. 439-582. Springer, 1999. http://dx.doi.org/10.1007/978-3-662-03882-6_5.

[52] M.N. Spijker. Stepsize conditions for general monotonicity in numerical initial value problems. SIAM J. Numer. Anal., 45(3):1226-1245, 2007. http://dx.doi.org/10.1137/060661739.

[53] R.J. Spiteri and S.J. Ruuth. A new class of optimal high-order strong-stabilitypreserving time discretization methods. SIAM J. Numer. Anal., 40(2):469-491, 2002. http://dx.doi.org/10.1137/S0036142901389025.

[54] C. Zhang and S. Vandewalle. General linear methods for Volterra integrodifferential equations with memory. SIAM J. Sci. Comput., 27(6):2010-2031, 2006. http://dx.doi.org/10.1137/040607058. 